\section{Weiterführende Literatur}

1. Feltgen N, Callizo J, Hattenbach L-O, Hoerauf $\mathrm{H}$ (2020) Dringlichkeit der operativen Versorgung bei der rissbedingten Netzhautablösung. Ophthalmologe. https://doi.org/10.1007/s00347-02001191-6

2. Callizo J et al (2017) Risk of progression in maculaon rhegmatogenous retinal detachment. Graefes Arch Clin Exp Ophthalmol 255(8):1559-1564

3. Di Lauro $S$ et al (2015) Loss of visual acuity after successful surgery formacula-on rhegmatogenous retinal detachment in a prospective multicentre study. J Ophthalmol 2015:821864

4. Diederen RM et al (2007) Scleral buckling surgery after macula-off retinal detachment: worse visual outcome after more than 6 days. Ophthalmology 114(4):705-709

5. Ehrlich R et al (2013) Timing of acute maculaon rhegmatogenous retinal detachment repair. Retina 33(1):105-110

6. Feltgen $\mathrm{N}$ et al (2013) Scleral buckling versus primary vitrectomy in rhegmatogenous retinal detachment study (SPR study): risk assessment of anatomical outcome. SPR study report no. 7. Acta Ophthalmol 91(3):282-287

7. Hajari JN et al (2014) Progression of foveolaon rhegmatogenous retinal detachment. $\mathrm{Br} J$ Ophthalmol 98(11):1534-1538

8. Heimann $\mathrm{H}$ et al (2007) Scleral buckling versus primary vitrectomy in rhegmatogenous retinal detachment: a prospective randomized multicenter clinical study. Ophthalmology 114(12):2142-2154

9. Henrich PB et al (2009) Macula-off retinal detachment-a matter of time? Klin Monbl Augenheilkd 226(4):289-293

10. Jackson TL et al (2014) United Kingdom national ophthalmology database study of vitreoretinal surgery: report 3, retinal detachment.Ophthalmology 121(3):643-648

11. Kontos A, Williamson TH (2017) Rate and risk factors for the conversion of fovea-on to fovea-off rhegmatogenous retinal detachment while awaiting surgery. Br J Ophthalmol 101(8):1011-1015

12. Koch KR et al (2012) Success rates of retinal detachment surgery: routine versus emergency setting. Graefes Arch Clin Exp Ophthalmol 250(12):1731-1736

13. Lai MM et al (2011) Anatomic and visual outcomes in early versus late macula-on primary retinal detachment repair. Retina 31(1):93-98

14. Sallam AB et al (2018) Royal college of ophthalmologists' national database study of vitreoretinal surgery: report 7, intersurgeon variations in primary rhegmatogenous retinal detachment failure. Retina 38(2):334-342

\title{
Erratum
}

Ophthalmologe $2021 \cdot 118: 238$

https://doi.org/10.1007/s00347-021-01347-y

Online publiziert: 15. Februar 2021

(c) Springer Medizin Verlag GmbH, ein Teil von Springer Nature 2021, korrigierte Publikation 2021

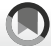

Check for
updates

Deutsche Ophthalmologische Gesellschaft (DOG) ${ }^{1} \cdot$ Retinologische Gesellschaft e. V. $(R G)^{2} \cdot$ Berufsverband der Augenärzte Deutschlands e. V. (BVA) ${ }^{3}$

'Deutsche Ophthalmologische Gesellschaft, München, Deutschland

${ }^{2}$ Retinologische Gesellschaft e. V., Freiburg, Deutschland

${ }^{3}$ Berufsverband der Augenärzte Deutschlands e.V., Düsseldorf, Deutschland

\section{Erratum zu: Stellungnahme von DOG, RG und BVA zur zeitlichen Planung der Versorgung einer rhegmatogenen Amotio retinae. Stand Oktober 2020}

\section{Erratum zu:}

Ophthalmologe 2021

https://doi.org/10.1007/s00347-020-

01311-2

In der ursprünglich veröffentlichten Version des Artikels war der Interessenkonflikt von Herrn Prof. Dr. Siegfried Priglinger unvollständig angegeben. Die Online-Version sowie das PDF des Beitrags wurden nachträglich korrigiert.

Wir bitten, den aktualisierten Beitrag zu beachten und den Fehler zu entschuldigen.

Die Redaktion

\section{Korrespondenzadresse}

Deutsche Ophthalmologische Gesellschaft (DOG)

Deutsche Ophthalmologische Gesellschaft Platenstr. 1, 80336 München, Deutschland geschaeftsstelle@dog.org
Die Online-Version des Originalartikels ist unter https://doi.org/10.1007/s00347-020-01311-2 zufinden. 